Journal of

\title{
Study of Non-Linear Deformation of Peripheral Stent Mechanics Using Com- putational Approach
}

\author{
Hua Yang ${ }^{1}$, Aleksandra Fortier ${ }^{1, *}, K_{\text {Kle Horne }}^{1}$, Tre Welch ${ }^{2}$, Atif Mohammad ${ }^{3}$, Subhash Banerjee ${ }^{3}$ \\ ${ }^{1}$ Mechanical and Energy Engineering Department, College of Engineering, University of North Texas, Denton, TX, \\ 76207 USA \\ ${ }^{2}$ Cardio Thoracic Surgery Department, UT Southwestern Medical Center Dallas, TX, 75390, USA \\ ${ }^{3}$ Department of Internal Medicine, UT Southwestern Medical Center and VA North Texas Health Care System, Dal- \\ las, TX, 75390, USA
}

${ }^{\star}$ Corresponding Author: Aleksandra Fortier, Mechanical and Energy Engineering Department, University of North Texas, Denton, TX 76207, USA. E-mail: drafortier@gmail.com

Received Date: August 24, 2016, Accepted Date: October 18, 2016 Published Date: October 22, 2016

Citation: Hua Yang (2016) Study of Non-Linear Deformation of Peripheral Stent Mechanics Using Computational Approach. J Biomed Eng 1: 1-10.

\begin{abstract}
Stent implants, specifically those implanted in Superficial Femoral Artery (SFA) in the lower limbs, are prone to high failure rates. The failures can occur due to abnormal behavior of the blood flow, mechanical forces from the arterial wall imposed on the stent, as well as leg movements such as bending, torsion, compression, and elongation that can create dynamic arterial environment imposed on the stent. In order to prevent high failure rates of peripheral stents there is a need to understand the unique biomechanical environment and the characteristics of the SFA arterial segment where stent is implanted. This study presents several steps that need to be taken into consideration before the complex and combined stent-artery-environment model can be created and analyzed. Results show finite element computational approach that demonstrates stent characteristics upon insertion inside the artery, creation of arterial segment with geometry and characteristics from real patient data, and theoretical approach to accurately impose blood flow onto the artery-stent model. This study recommends procedure for analyzing biomechanical environment of the stent-artery system using real artery geometry. Results show that stent design and artery specific geometry play a critical role in stent performance.
\end{abstract}

Keywords: Peripheral stent; Mechanical behavior; Non-linear deformation; Finite elements modeling; Stent-artery system; Restenosis

\section{Introduction}

Cardiovascular disease is often related to atherosclerosis which can cause narrowing, rupture or erosion of the arterial wall, with eventual reduction (i.e. $>50 \%$ blockage) or occlusion (i.e. $100 \%$ blockage) of blood flow [1]. Among several different treatments is stent implant procedure, which is the insertion of a metal and/or plastic tubular structure that has the ability to expand into a cylindrical shape, either by use of balloon or a self-expansion mechanism $[1,2]$. It is reported that about 700,000 stent procedures (in coronary and peripheral arteries together) are done in the U.S. annually with about $50 \%$ stent failure rate in the peripheral arteries reported [3].

C 2016 The Authors. Published by the JScholar under the terms of the Creative Commons Attribution License http://creativecommons.org/licenses/ by/3.0/, which permits unrestricted use, provided the original author and source are credited.
High failure rate of stent implants is a result of complex dynamic environment when delivered to a region of complex peripheral artery anatomy. Finite element analysis and numerical simulation tests are increasingly important for accurate design and manufacturing of stents in order to meet market demands and improve patient care. The possibilities of Finite Element Analysis (FEA) and Computational Fluid Dynamics (CFD), combined with contemporary imaging techniques, greatly facilitate stent research. Tremendous amount of research has been done on coronary artery stenting, on stent geometry, and on estimating artery-stent interaction however, very little is reported on superficial artery stenting and the process of using real artery geometry from patient data for the computational models [4-7]. 
Most studies estimate the geometry of the artery as cylinder with uniform diameter across its length where in reality arteries have curves and tortuosity specific to the patient anatomy $[1,2,4]$. Several aspects need to be considered in order for computational models to accurately estimate the artery-stent biomechanical environment and the issues causing stent failure. Stent geometry and loading conditions need to be accurately defined using principles of solid mechanics [8]. The real artery geometry is typically obtained by using Computed Tomography (CT) images, or intravascular ultrasound (IVUS) images, and reconstructing them using the Mimics Innovation Suite Software that will give $3 \mathrm{D}$ artery model with real geometry [9]. The constructed 3D model is the transferred to finite elements modeling software package coupled with stent model and various analyses are performed. To complete the numerical analysis especially the nonlinear analysis, the finite element method requires a lot of information, including the geometry and the material properties of the stents, the effect of the catheter and the artery, and the accurate application of boundary and loading conditions. MSC commercial software allows for proper analysis of these inherently difficult (e.g. non-homogeneous, non-linear) 3D models [10]. In our previous study [2] we have used MSC Software to validate the linear deformation of specific stent designs also analyzed theoretically and here we use similar computational approach to simulate nonlinear deformation behavior of the stent. In this study the appropriate finite element models are developed in Patran and solved with NASTRAN [11]. Prevalent stents have numerous design patterns, the widespread interest focused on the properties of stent designs in terms of multiple types geometry $[1,5,6]$. The elevated stress region largely depends on the strut shape of stents in terms of mechanical stress corresponding to the clinical observations $[12,13]$. The results from this study show that the strut length, separation angle and cell asymmetry are crucial to the performance of stent design. Finite element modeling approach is used to evaluate the deformation in balloon expanded stent under internal pressure first. Results from this study show the nonlinear behavior of the stent, and the plastic region with the maximum equivalent stress noted to occur at the 4.5 millimeter ( $\mathrm{mm}$ ) expansion diameter. Approach for generating artery 3D models from real patient data and imposing dynamic blood flow behavior is also presented. A fundamental understanding and step by step process for generating stent-artery-environment models have limited reports in the literature published to date.

\section{Materials and Methods}

\section{Stent Modeling and Deformation Analysis Meth-}

od: Stent expansion is analyzed assuming struts within the stent expand at a constant rate via a plastic hinge mechanism. For each single hinge-strut unit, the beam theory (explained in prior study [2]) continuously contributes to the understanding of the complex and nonlinear inflation of expandable stent. The finite element model estimates and reproduces the characteristics during crimp and inflation mechanism. In the stent model, the stent geometry consists of several rows of identical parts, one intermediate row at each end (the same geometry, but different strut width), and one final row at each end that has the same basic geometry (and increased strut width) with the exception that there is no 'connector' on the outboard ends (as there are no additional rows to be attached to).
The repetitive nature of the geometry allows for a theoretical model or a finite element model of a portion of the entire stent to accurately characterize the stress state within the entire stent when inflated. This approach allows the finite element mesh to be finer in the region that is modeled, as opposed to using a similar number of elements for an entire stent. For example, the assumed strain formulation allows the element to accurately capture bending characteristics with as little as one element through the thickness. Similarly, in this study is assumed that due to symmetry unit structure analysis will reflect the deformation of the entire stent, where expansion, deflection and stress/strain will be also predicted. $3 \mathrm{~mm}$ diameter stent is inflated to $4.5 \mathrm{~mm}$ which produces large mechanical loads with nonlinear characteristics and these are considered in our model. In real application, initially the stent is an empty and collapsed balloon loaded on a guide wire, known as a balloon catheter, the combo is passed into the narrowed arteries and then inflated to a $4.5 \mathrm{~mm}$ using pressures approximately 75 to 500 times normal blood pressure [14]. Additionally, most healthy arteries yield an average hoop stress of approximately $100 \mathrm{KPa}$ which gives an idea what is the stent undergoing once placed inside the artery. By simulating the inflated stent structure, the maximum stress will be evaluated as a result of stent inflation to diameter of up to $4.5 \mathrm{~mm}$. A Von Mises yield condition, commonly used with metallic alloys, along with a kinematic hardening law are used in the analysis. When the material is strained beyond the yield point, the yield surface must change for continued strain hardening. The kinematic hardening law is used for the analysis herein, to take into account the Bauschinger effect present during reversal of the stress field which occurs between the compression and inflation stages. Bauschinger effect describes that pre-straining in any direction, as defined by the principal axis of the strain tensor, will introduce an anisotropy for further deformation in any other direction [15]. The intensity of this pre-strain associated anisotropy is at maximum when the direction of further straining is opposite to that of the pre-strain. A finite element test model was also created in order to verify that the elastic-plastic material model used in the analysis accurately represents the supplied material data. Generally, inflation of the stent may be performed using direct pressure applied to inside diameter of the stent or manually expanding the stent through prescribed boundary conditions. Using MSC.NASTRAN nonlinear finite element analysis (implicit solution) (MSC. Software. Nastran, CA, USA) will analyze the stent with rhombus structure shown in Figure 1.

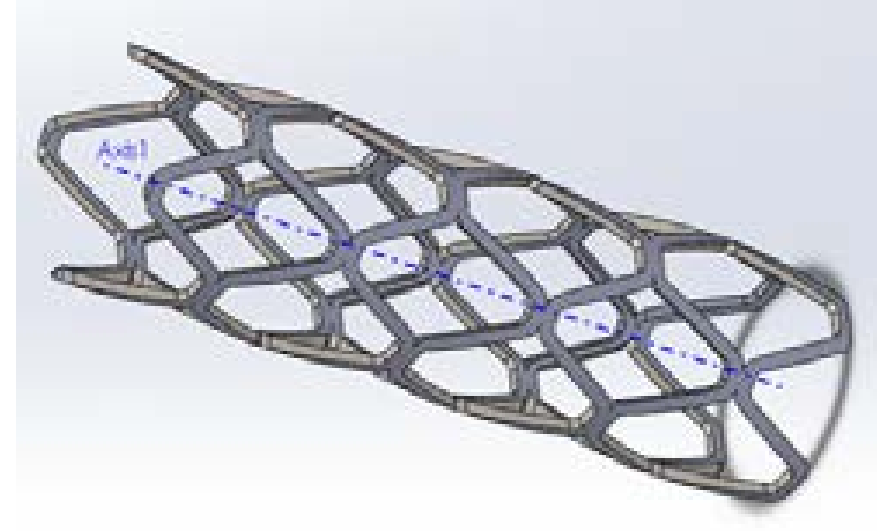

Figure 1: 3D model of 8 -sturt structure stent with 4 rhombus cross-sectional units 
In the model plastic hinge will be imposed at each connecting joint. Stent expansion is analyzed assuming struts within the stent will expand at a constant rate via a plastic hinge mechanism. The strut unit is the analytical model considered and illustrated as presented in Figure 2.

a)
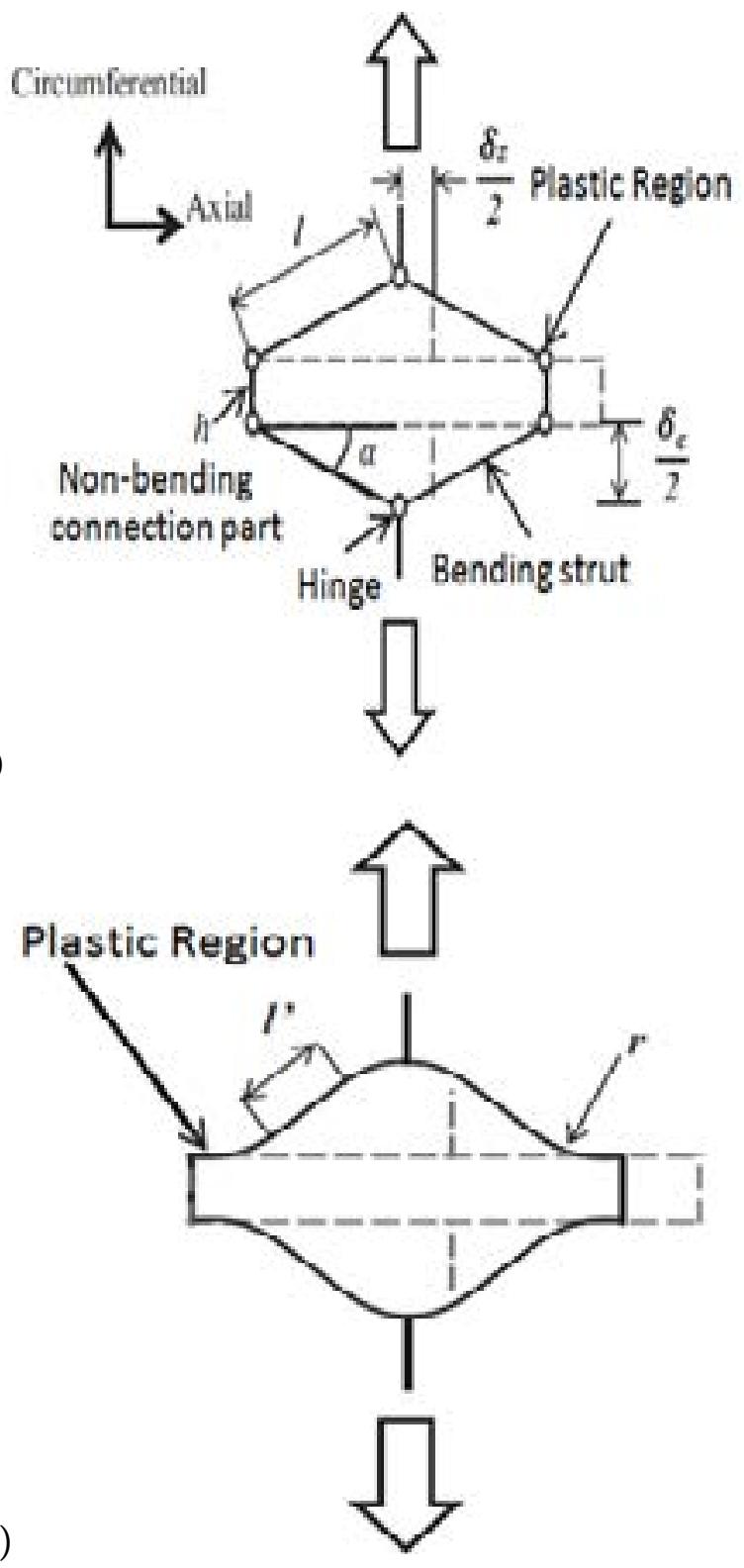

Figure 2: Nonlinear analysis approach for stent with rhombus structure considering the connection part of model a) idealized analytical model in non-linear analyses b) part curvature of plasticity region due to the expansion forces [16].

The modeling approach of expansion considers a bending stent strut as pairs of cantilevers loaded by a point force at the connection part, applied perpendicular to the initial cantilever position. During the expansion procedure, the eight or sixteen struts (depending on the stent in question) in a circle grow along the sphere are viewed as bending struts [15]. The circumferentially connecting parts are deformed without bending. Then the stent expands $\delta_{-} z$ in the axial direction and $\delta_{-} c$ in the circumferential direction (Figure 2a). Furthermore, the connection region with plastic hinge on the struts represents radius of curvature $(r)$ and the deformed length of strut $\left(1^{\wedge^{\prime}}\right)$ in the plastic region (Figure $2 b$ ).
The analysis of the stent inflation requires a model capable of handling large deformations and large strains. In addition, nonlinear material properties must be considered to accurately capture the deformation process. Therefore, the Lagrangian and the Eulerian descriptions are considered for the large deformations of the stents [17]. The Lagrangian or material approach uses the initial coordinates of each particle to describe the deformation whereas the Eulerian or spatial description uses the coordinates of the particles in the deformed state to describe the deformation, when elasticity effects is prominent [17]. Here we are using the Lagrangian approach. The stress and strain calculations are based upon true (Cauchy) stress and true (logarithmic) strain measures [8]. The axial stress, circumstantial stress and radial stress are considered present under anatomical bending. The artery wall pressure depends on the blood properties. Arterial pressure of the systemic circulation in terms of the systolic pressure over diastolic pressure is represented as compressive stress [20]. During the nonlinear analysis, Lagrangian method is considered with respect to the un-deformed configuration. The inflation of the stent which causes large deformation is modeled using an elastic-plastic material model, and inelastic strains. Solution of nonlinear equations, ${ }^{\mathrm{t}+\Delta t} \mathrm{R}$ is externally applied loads, ${ }^{\mathrm{t}+\Delta t} \mathrm{~F}$ is nodal point forces corresponding to internal element stresses presented by the relationship in equation 1 [17]:

${ }^{t+\Delta t} R-{ }^{t+\Delta t} F=0$

Equation 1 is set to equal equation 2 through the relationship: ${ }_{0}{ }^{t} \mathrm{k} \cdot \Delta \mathrm{u}={ }^{\mathrm{t}+\Delta \mathrm{t}} \mathrm{R}-{ }^{\mathrm{t}+\Delta \mathrm{t}} \mathrm{F}^{(\mathrm{i}+1)}=0$

where the iteration, $\mathrm{i}=1,2,3, \ldots{ }_{0}^{\mathrm{t}} \mathrm{k}$ means the stiffness matrix of the structure at initial state, and $\Delta \mathrm{u}$ is the displacement. At convergence, the compatibility is satisfied with stress-strain law and equilibrium of nodal point and local force resulting in the following equilibrium equation 3 :

$$
{ }^{t+\Delta t} \mathrm{R}={ }^{\mathrm{t}+\Delta \mathrm{t}} \mathrm{F}
$$

This relates to the updated Lagrangian method being used during inelastic deformations, and the stress and strain calculations are based upon true (Cauchy) stress and true (logarithmic) strain measures. The 3D model is meshed with quadratic 10-noded tetrahedral elements (TETRA10). Approximate mesh size $=0.001 \mathrm{~mm}$ with five or six elements across the strut width is defined. Total number of elements 35,777 and total number of nodes 100,113 for the 3D stent model analyzed. Strut angle $20^{\circ}$. The stent material is $316 \mathrm{~L}$ stainless steel with properties listed in table 1 . . Boundary conditions are applied at each end. One end fixed which allows for axial displacement and the line along the total length of the stent, constrains displacement in transverse direction. The other end is free. $83 \mathrm{KPa}$ pressure is the load which is assumed upon stent being inserted in the artery and inflated to a maximum diameter of $4.5 \mathrm{~mm}$. This is done by pressurization of an elastomeric balloon material inserted inside the tube (i.e. stent) which mimics the inflation activity. 


\begin{tabular}{|l|l|}
\hline Property & Value \\
\hline Young's modulus & $190 \mathrm{GPa}$ \\
\hline Poisson ratio & 0.3 \\
\hline Yield stress & $207 \mathrm{MPa}$ \\
\hline Ultimate stress & $515 \mathrm{MPa}$ \\
\hline Limit nominal strain & $60 \%$ \\
\hline
\end{tabular}

Table 1: Materials properties of 316L SS stents

Artery and Blood Flow Modeling Approach: Despite that stent implants are efficient in restoring normal blood flow over time new blockage can form called restenosis [1]. Research studies based on clinical data have concluded that in addition to stent design, the geometry of the artery can contribute to the development of restenosis $[4,21,22]$. For example several conditions such as twisting, compression, elongation and flexion as shown in Figure 3 can be noted for artery. These conditions contribute to the stent deformation and performance over time and this is why mirroring real artery geometry for modeling accuracy is of great importance.

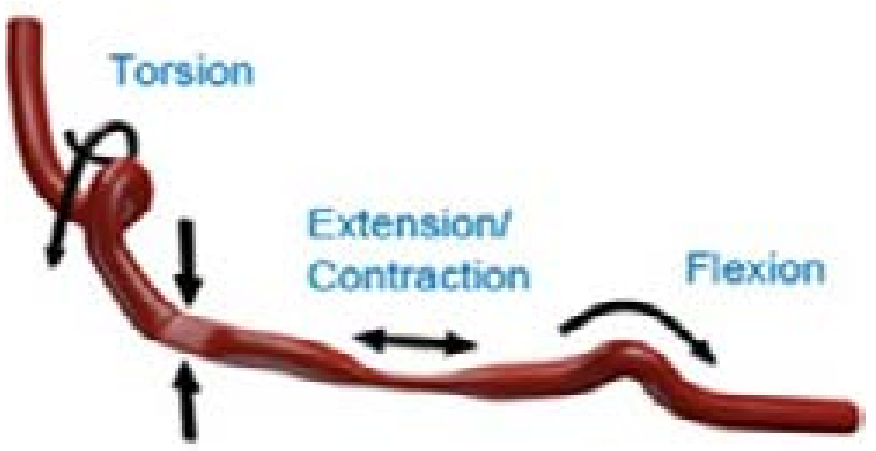

\section{Compression}

Figure 3: Non-uniform artery geometry [1]

In this study IVUS images were obtained from UT Southwestern Medical Center in Dallas, Texas for a patient with specific conditions and using slicing function and custom developed code at UNT 3D artery model was constructed along with the type of blood flow in the specific segment of the artery as shown in Figure 4. We can see from Figure 4 that the real artery has special contours and curves along its walls that vary from typical cylindrical shapes with uniform geometry used in many modeling studies.

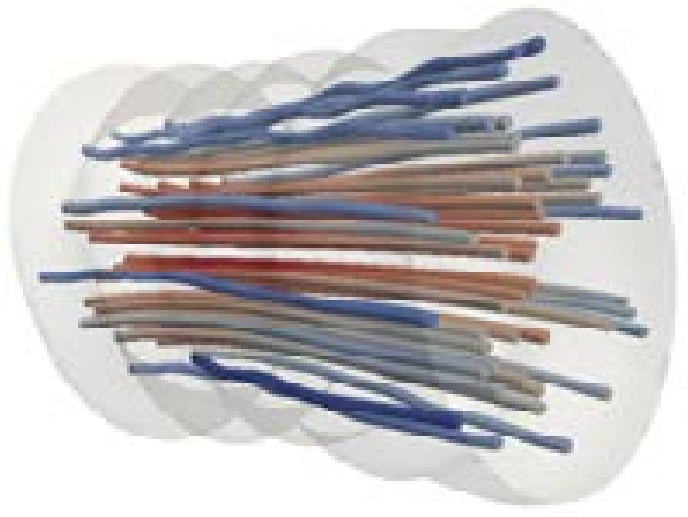

Figure 4: 3D artery model with blood flow developed from IVUS image
The rheological behavior of blood is quantified by non-Newtonian viscosity. The computational blood flow model is taken as three-dimensional and transient flow in deforming blood vessels. Newtonian fluid viscosity is always constant against the shear rate. By comparison, non-Newtonian fluid viscosity changes depending on the shear rate. Literature demonstrates the relationship between blood viscosity and the shear rate for Newtonian and four different non-Newtonian models [23]. For our approach popular Carreau non-Newtonian model [23] is selected with features zero-shear plateau at low rates and a power-law region at high rates as demonstrated by the equation 4:

$\mu(|\gamma|)=\mu_{\infty}+\left(\mu_{0}-\mu_{\infty}\right)\left[1+(\lambda \gamma)^{2}\right]^{(\mathrm{n}-1) / 2)}$

$\mu_{0}=0.056 \mathrm{~Pa} \cdot \mathrm{s}$ viscosity at zero shear rate,

$\lambda=3.131$ time constant, and

$\mathrm{n}=0.3568$ power law index.

Reynolds number is set as 300 for incompressible, homogeneous and sinusoidal pulsatile flow and artery wall is viewed as rigid body. The Carreau model describes pseudoplastic flow with asymptotic viscosities at zero $\mu_{0}$ (Newtonian) and infinite $\mu_{\infty}(n<1$ non-Newtonian $)$ shear rates and with no yield stress; $\lambda$ is a constant with units of time, after $\lambda=1 / \gamma$, non-Newtonian behavior is important; $n=0.3568$ is power law index as a measure of the deviation of the fluid form Newtonian ( $n=1$, Newtonnian fluid; $n>1$, shear-thicking(dilatant fluid); $\mathrm{n}<1$, shearthinning (pseudo-plastics)) (Ewoldt). Following steps $1-7$ in supplementary data section the average velocity and the shear stress are derived in the control volume.

\section{Results}

Figure 5 shows results of expanded stent as the balloon is inflated, and each of the struts open.

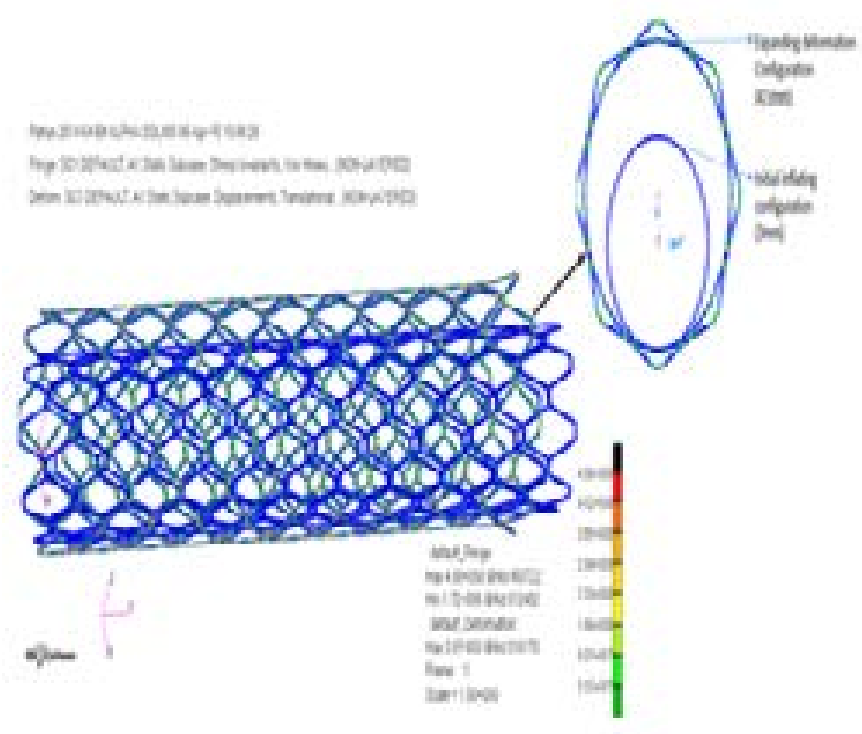

Figure 5 - Stress distribution of inflated stent under pressure from $3 \mathrm{~mm}$ (blue configuration) to $4.5 \mathrm{~mm}$ (green configuration) 
The expandable stage is noted by the green configuration and when the stent is released and allowed to spring back to a 'free state' noted by the blue configuration. Cross-sectional view of expanded versus initial diameter is shown in the upper right corner of Figure 5. The equivalent stress was calculated to be around 498MPa shown on the contour of model at around 50\% expansion in diameter (i.e. from $3.0 \mathrm{~mm}$ to $4.5 \mathrm{~mm}$ ).

Figure 6 illustrates close view of the expanded configuration with respect to the maximum equivalent stress value of the structure, of around $498 \mathrm{MPa}$. The plastic regions (shown with yellow and green color in Figure 6) are concentrated along each hinge joint as expected and predicted by the analytical model shown in Figure $2 \mathrm{~b}$ and again in Figure 6 to the right.

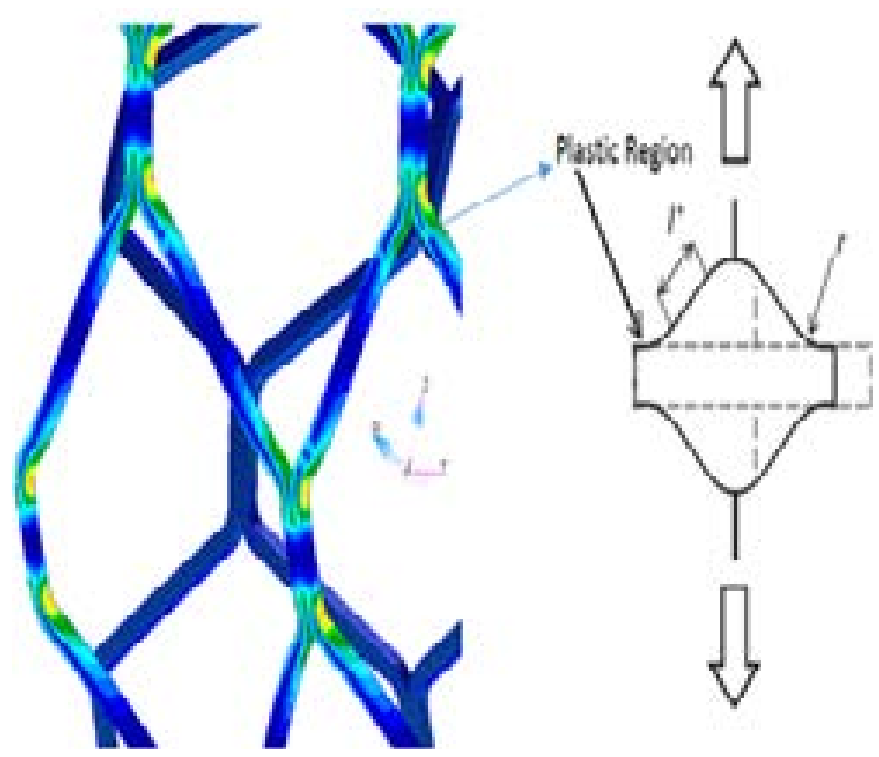

Figure 6: Stent configuration after inflation showing the plastic regions with the maximum equivalent stress presented with yellow/green colors and aligns with the theoretical model

Using rheological equations of blood (presented as equations 4 in this paper and 1-7 from supplementary data section), the viscosity versus shear rate relationship is predicted by calculating the following parametric values summarized in Table 2.

\begin{tabular}{|l|l|}
\hline Parameter & Calculated Value \\
\hline $\begin{array}{l}\text { Power law index in Carreau } \\
\text { model }(\mathrm{n})\end{array}$ & 0.344 \\
\hline Zero shear rate limit $\left(\mu \_0\right)$ & $0.0456 \mathrm{~Pa} \cdot \mathrm{s}$ \\
\hline $\begin{array}{l}\text { Infinite shear rate limit } \\
\left(\mu \_\infty\right)\end{array}$ & $0.0032 \mathrm{~Pa} \cdot \mathrm{s}$ \\
\hline Relaxation time constant $(\lambda)$ & $10.03 \mathrm{~s}$ \\
\hline Ideal mean velocity of blood & $0.35 \mathrm{~m} / \mathrm{s}$ \\
\hline
\end{tabular}

Table 2: Parametric values for the non-Newtonian constitute equations

Figure 7a illustrates the velocity distribution of the blood flow in artery cross-section and the maximum velocity at the centerline of the artery where the contour showing approximately $0.685 \mathrm{~m} / \mathrm{s}$. The blood viscosity distribution in the artery is also shown in Figure $7 \mathrm{~b}$.
Figure $7 \mathrm{c}$ illustrates calculated pressure gradient value of approximately $1.5 \mathrm{kPa} / \mathrm{m}$ to correspond to the ideal mean velocity of approximately $0.35 \mathrm{~m} / \mathrm{s}$ also listed in Table 2 .

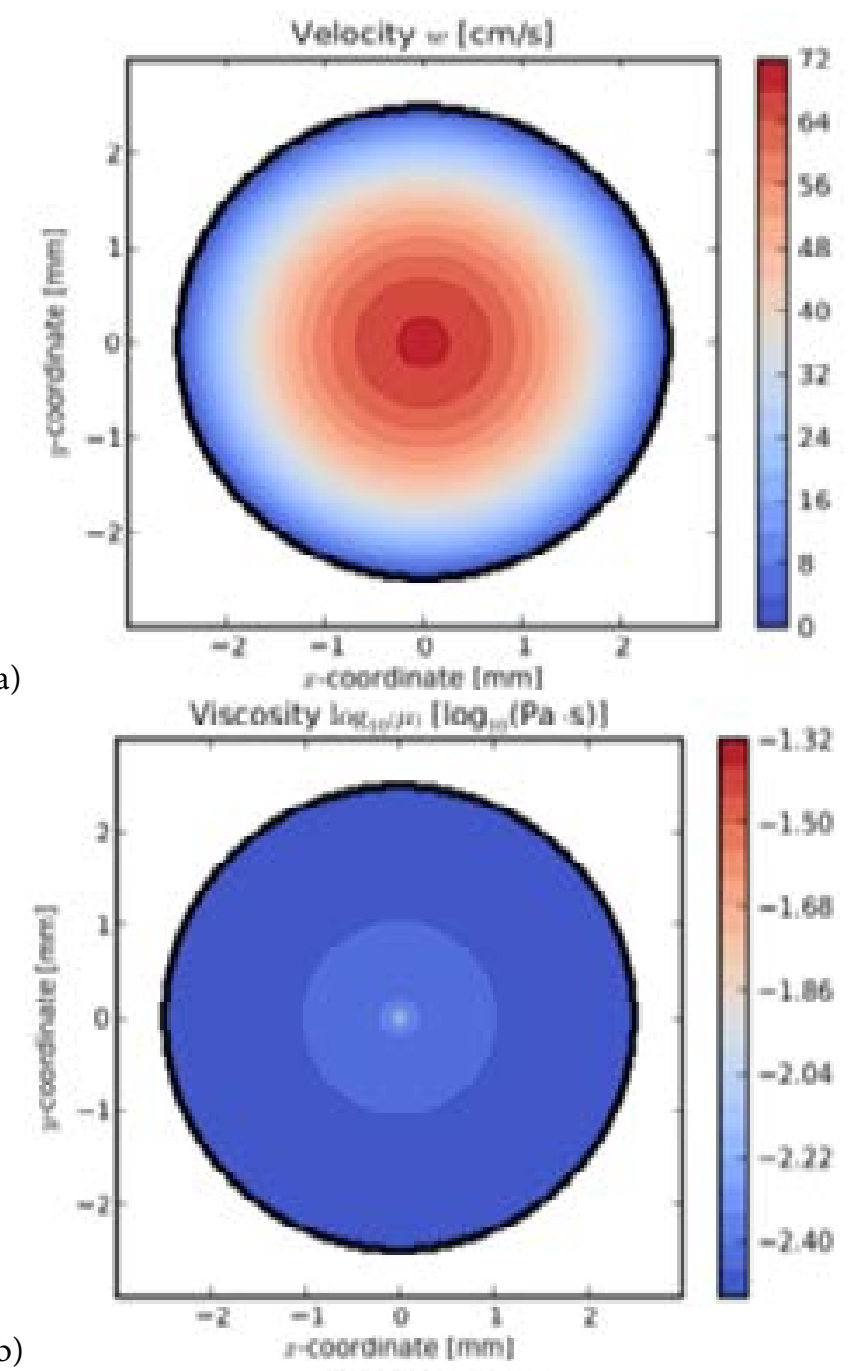

b)

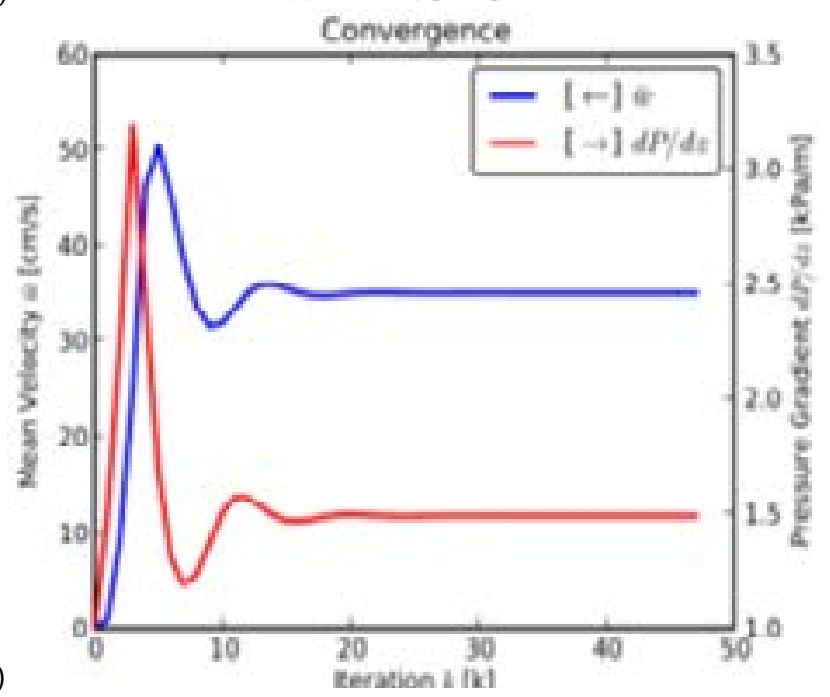

Figure 7: a) Velocity distribution of the blood flow in artery cross-section b) viscosity distribution of the blood flow in artery cross-section c) Comparison of the mean velocity and pressure gradient over several iterations 


\section{Discussions}

During stent deformation analysis it is noticed that the dominant change of loading appears to cause an increase tensile load in the connection parts. The stress is primarily caused as a result of bending as the struts open and straighten out during stent inflation. The maximum equivalent stress of $498 \mathrm{MPa}$ occurred at the $4.5 \mathrm{~mm}$ expansion diameter and was found to be less than the stainless steel ultimate strength of $515 \mathrm{MPa}$ indicating a safe stent design throughout the expansion range. The true-stress values are typically larger than the respective engineering stress measured values, which is commonly used with material test data. This must be considered when comparison of maximum stress results reported in this study are to be made with engineering test data. Generally, the experimental data from a uniaxial tension test is expressed in terms of true stress vs. true strain, not engineering stress or strain. The maximum stress values were found to occur in localized regions of the stent. These regions were generally found along the inner radii of each of the 'corner' links connecting each of the longitudinal struts. Stress values throughout the whole stent were typically much lower. As to be expected, the stress in each of the radius sections exceeded the elastic limit of the material $(207 \mathrm{MPa})$, resulting in plastic (or permanent) deformation of the stent upon removal of the loading. If the stress not exceeded the elastic limit, removal of the load would result in the stent recovering its initial configuration. It was also noticed that the apparent blood viscosity decreases as the flow rate increases. The greater the flow is, the greater the rate is where one lamina of blood shears against an adjacent lamina of blood. The greater tendency for blood accumulation in the axial lamina at higher flow rates is partly responsible for this non-Newtonian behavior. However, it is possible that at very slow flow rates, the suspended cells tend to form aggregates, which increases blood viscosity. As flow is increased, this aggregation decreases, and so does the viscosity of blood.

In summary, the study demonstrated that the geometric parameters and structure can play main factors in the performance of the stent implant. A fundamental understanding of the mechanics of the unit shape involving compression/inflation activity and their modeling analysis give important insights for optimize stent designs. Combination of clinical performance reports with FEA modeling approach can simulate future real physical situation, using refined numerical studies and more complex cases such as stent-artery or stent-flow interactions. Using the insights gained from this study will allow us to analyze stent mechanical behavior and life-cycle under artery and blood flow effect for future cases. The future work can lead into personalized patient care where computational models can match stent configuration and material suitable with the patient disease and health state.

\section{Acknowledgements}

This work was supported by internal funds from UNT. We thank the team at UTSW Medical Center for providing valuable feedback and stent samples for analyses as well as IVUS images.

\section{Supplementary Data}

The rate-of-deformation tensor $\mathrm{D}$ and the shear rate $\gamma$ :

$$
\begin{aligned}
& D=1 / 2\left(\left(\partial u \_j\right) /\left(\partial x \_i\right)+\left(\partial u \_i\right) /\left(\partial x \_j\right)\right) \\
& \gamma=\sqrt{ }(2 D: D)
\end{aligned}
$$

The shear stress is simply written in terms of a non-Newtonian viscosity $\mu(\gamma)$ :

$\tau=\mu(\gamma) \mathrm{D}$

In Carreau model, considering $\mathrm{u}=0, \mathrm{v}=0$, velocity won't change, $\partial \mathrm{u} / \partial \mathrm{x}=0, \partial \mathrm{v} / \partial \mathrm{y}=0, \partial \mathrm{w} / \partial \mathrm{z}=0$.

Then, the momentum balance equation becomes:

$\rho(\partial \mathrm{v}) / \partial \mathrm{t}+\rho(\mathrm{v} \cdot) \mathrm{v}=-\mathrm{P}+\cdot \tau$

Momentum in $\mathrm{x}$-direction is derived: $\rho u \partial u / \partial x+\rho v \partial u / \partial y=-\partial P / \partial x+\partial / \partial x(\mu \partial u / \partial x)+\partial / \partial y(\mu \partial u / \partial y)$

Momentum in $y$-direction is derived: $\rho u \partial v / \partial x+\rho v \partial v / \partial y=-\partial P / \partial y+\partial / \partial x(\mu \partial v / \partial x)+\partial / \partial y(\mu \partial v / \partial y)$

Momentum in $\mathrm{z}$-direction is derived: $\rho u \partial w / \partial x+\rho v \partial w / \partial y=-\partial P / \partial z+\partial / \partial x(\mu \partial w / \partial x)+\partial / \partial y(\mu \partial w / \partial y)$

$\partial \mathrm{P} / \partial \mathrm{z} \neq 0$ Then, equation is simplified as: $\partial \mathrm{P} / \partial \mathrm{z}=\partial / \partial \mathrm{x}(\mu \partial \mathrm{w} / \partial \mathrm{x})+\partial / \partial \mathrm{y}(\mu \partial \mathrm{w} / \partial \mathrm{y})$

$\gamma=\sqrt{ }\left((\partial w / \partial x)^{\wedge} 2+(\partial w / \partial y)^{\wedge} 2\right)$

Then, to compute the blood flow through two ends constricted artery, the solver is implemented to solve sequentially the momentum balance equation. The coupling between velocity and pressure drop is achieved through algorithm. The continuity control volume (i.e. cross-section of the artery) is analyzed as discretized domain shown in Figure 1a. For 2D model, the viscosity at the control volume faces is evaluated precisely by setting the grid points and draw control volume faces between neighboring points as shown in Figure $1 \mathrm{~b}$.

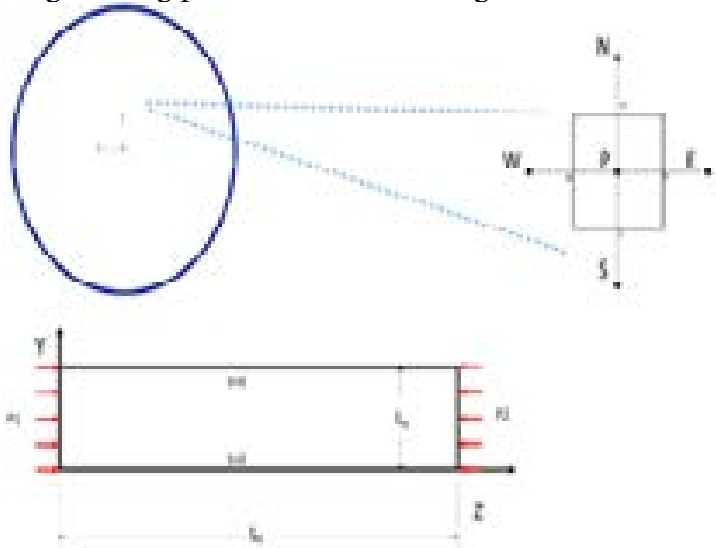

Figure 1: a) Segment of blood model field (cross-section of the artery) as the control volume of studied flow 

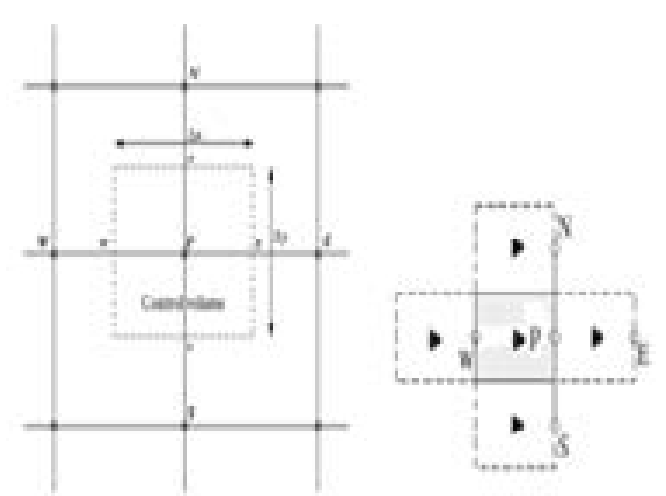

Figure 1: b) finite volume cell with neighbor points in the control volume

Generally, the viscosity at $\mathrm{n}$ point can be interpolated as equation (6):

$\mu_{\mathrm{n}}=\left(2 \mu_{\mathrm{N}} \cdot \mu_{\mathrm{P}}\right) /\left(\mu_{\mathrm{N}}+\mu_{\mathrm{P}}\right)$

Then the average velocity and the shear stress are derived in the control volume as:

$\mathrm{w}=\left(\int_{\mathrm{cv}} \mathrm{wdA}\right) /\left(\int_{\mathrm{cv}} \mathrm{dA}\right) \quad \mathrm{F}_{\text {shear }}=\int \mu \mathrm{dw} / \mathrm{dn} \mathrm{dl} \quad \mathrm{n}=\mathrm{n}, \mathrm{s}, \mathrm{w}, \mathrm{e}$

\section{References}

1) A Fortier, V Gullapalli, R A Mirshams (2014) Review of Biomechanical Studies of Arteries and TheirEffect on Stent Performance. International Journal of Cardiology 4: 12-18.

2) H Yang (2015) Study Of Mechanical Performance of Stent Implants Using Theoretical \& Numerical Approach.Master's Thesis, Denton, TX.

3) Banerjee S, Das TS, Abu-Fadel MS, Dippel EJ, Shammas NW, Tran DL, et al (2012) Pilot Trial of Cryoplasty or Conventional Balloon Post-Dilation ofNitinol Stents for Revascularization of Peripheral Arterial Segments-The COBRA Trial. J Am Coll Cardiol 60:1352-1359. 4) Ansari F, Pack LK, Brooks SS, Morrison TM (2013) Design Considerations for Studies of the Biomechanical Environment of the Femoropopliteal Arteries. Journal of Vascular Surgery58: 804-813.

5) Garg S, Serruys PW(2010) Coronary Stents Looking Forward. Journal of the American College of Cardiology 56: 0735-1097.

6) Garg S, Serruys PW (2010)"Coronary Stents: Current Status". J Am Coll Cardiol 56: 1-42.

7) Welch TR (2009) Advances in Helical Stent Design and Fabrication Thermal Treatment and Structural Interaction Studies of the Simulated Plaque-Laden Artery. Dissertation 6926-6227.

8) Steven R Schmid, Bernard J. Hamrock, Bo. O. Jacobson, (2004) Fundamentals of Machine Elements, 3rd , Prentice Hall.

9) http://biomedical.materialise.com/mis last accessed April 2016.

10) http://www.mscsoftware.com/en-asean/node/970 lastaccessed April 2016.

11) http://www.healthline.com/health/atherosclerosis last accessed April 2016.

12) L Gu, S Zhao (2012) Arterial Wall Mechanics and Clinical Implications after Coronary Stenting: Comparisons of Three Stent Designs. Int J Appl Mechanics 67-81.

13) Linxia Gu, Shijia Zhao, Stacey R. Froemming (2012) Arterial wall mechanics and clinical implication after coronary stenting: comparisons of three stent designs. Int J Appl Mechanics 4:14.

14) Timmins LH, Moreno MR, Meyer CA, Criscione JC, et al. (2007) Stented Artery Biomechanics and Device Design Optimization. Med Biol Eng Comput 45: 505-513.

15) Abel A (1982) Historical Perspectives and Some of the Main Features of the Bauschinger Effect, Mater. Forum10:11-26.

16) Douglas GR, Phani AS, Gagnon J (2014) Analyses and design of expansion mechanisms of balloon expandable vascular stents. J Biomech 6: 1438-1446.

17) Updated Lagrangian formulation for incremental general nonlinear analysis. MIT online course.

18) Paryab N, Cronin DS, Lee-Sullivan P (2014)Finite element methods to analyze helical stent expansion. Int J Numer Method Biomed Eng 30: 339-352.

19) Kiousis DE, Wulff AR, Holzapfel GA (2009) Experimental Studies and Numerical Analysis of the Inflation and Interaction of Vascular Balloon Catheter-Stent Systems.Ann Biomed Eng37: 315-330.

20) Nicholas Karnessis, Gaetano Burriesci (2013) Uniaxial and Buckling Mechanical Response of Auxetic Cellular Tubes. Smart Materials and Structures $22: 31$.

21) Glagov S, Zarins C, Giddens DP, Ku DN (1998) Hemodynamics and Atherosclerosis Insights and Perspectives Gained from Studies of Human Arteries. Arch Pathol Lab Med 1018-1031.

22) Timmins LH, Miller MW, Clubb FJ Jr, Moore JE Jr (2011) Increased Artery Wall Stress Post-Stenting Leads to Greater Intimal Thickening. Lab Inves 91: 955-967.

23) M G Rabby, S P Shupti,Md M Molla (2014) Pulsatile Non-Newtonian Laminar Blood Flows through Arterial Double Stenoses. Journal of Fluids 1-13. 
Submit your manuscript to a JScholar journal and benefit from:

q Convenient online submission

- Rigorous peer review

- Immediate publication on acceptance

- Open access: articles freely available online

I High visibility within the field

I Better discount for your subsequent articles

Submit your manuscript at http://www.jscholaronline.org/submit-manuscript.php 